\title{
EDITORIAL
}

\section{Profesores frente a estudiantes: las dos orillas de la educación bimodal}

\author{
Magíster Beatriz Eugenia Quiceno Castañeda \\ Magíster Sandra Isabel Arango Vásquez \\ Magíster Claudia Patricia Vásquez Lopera
}

\section{Resumen}

Desde hace 6 años el grupo de investigación E-Virtual de la Universidad de Medellín viene trabajando en la implementación de asignaturas bimodales en la Institución. En el 2009, con el apoyo de MEN, se implementó la modalidad a distancia con metodología virtual en el modelo pedagógico de la Universidad.

Estas nuevas experiencias llevaron al Grupo a cuestionarse sobre las características pedagógicas y didácticas a tener en cuenta cuando se combinan la educación presencial y la virtual. Para ello se indagó con profesores y estudiantes sobre su percepción al respecto. Para la recolección de información se combinaron técnicas cualitativas y cuantitativas, que han arrojado interesantes resultados, entre ellos proceso de inducción, interacciones comunicativas, Objetos Virtuales de Aprendizaje y uso de la plataforma virtual.

En este artículo se darán a conocer algunos resultados de la investigación, cuáles han sido los aspectos positivos de esta experiencia y cuáles son las áreas a mejorar.

Palabras clave: bimodal, educación, modelo didáctico, modelo pedagógico, E-learning. 


\section{Lecturers versus students: The two sides of blended education}

\section{ABSTRACT}

The research group E-Virtual from the Universidad de Medellin has been working on the implementation of bimodal courses since six years ago. In 2009, with the support of the Ministery of National Education (MEN), it was implemented the non-face-toface class modality with a virtual methodology in the pedagogic framework of the University. This new activities took the group to think about the didactic and pedagogic characteristics that should be considered when face-to-face and virtual education are combined. To achieve this, we asked lectures and students about the perception of this new methodology. The data was collected by combining qualitative and quantitative methods. As a result, we obtained an introductory process, communicative interactions, virtual learning objects, and clues about the use of the platform. In this paper we provide the preliminary results of our study, emphasizing on the positive aspects, and the aspects to improve.

Key words: bimodal, education, didactic model, pedagogic model, E-learning 
A continuación, se presentan algunos de los resultados de la investigación Características que deben tener las asignaturas bimodales en la Universidad de Medellín para definir los criterios generales requeridos para su implementación institucional, en concordancia con el modelo pedagógico de la Universidad.

Estos resultados hacen parte de uno de los objetivos de la investigación, el cual busca determinar desde la perspectiva de estudiantes y profesores los aspectos positivos y las áreas de mejora que deben ser tenidas en cuenta en la educación bimodal en la Universidad de Medellín.

\section{Metodología}

\section{La perspectiva de los estudiantes frente a la educación bimodal en la Universidad de Medellín}

Inicialmente se mostrarán los resultados desde el punto de vista de los estudiantes.

Entre los períodos académicos 2011-1 y 2012-2, las tres asignaturas bimodales ofrecidas en la Universidad de Medellín tuvieron un total de 434 estudiantes matriculados en 3 asignaturas diferentes: Electiva TIC (110 estudiantes), Mercadeo I (127 estudiantes) y Estética de la Imagen (197 estudiantes)

Para muchos de ellos, la experiencia con la asignatura bimodal fue su primer contacto con una nueva realidad en su proceso de formación profesional; otros ya habían tenido algún acercamiento con las TIC enfocadas a la educación, unos a mediante cursos que habían tomado de manera libre a través de la Web, otros en la plataforma E-learning de la Universidad, donde muchos profesores les dejaban lecturas y les solicitaban el envío de trabajos académicos.

Desde 2011-1 se empezaron a aplicar encuestas a los estudiantes al final de cada semestre para que expresaran sus opiniones sobre el proceso que habían llevado a cabo durante el curso de su asignatura bimodal.

Durante los 2 años de mediciones, el número de estudiantes que respondió el cuestionario fue de 173 de los 434 matriculados. Este número corresponde al 40 \% del total de estudiantes de 3 asignaturas diferentes, evaluados en 4 períodos académicos comprendidos entre $2011_{\text {_ }}{ }^{\text {y } 2012_{-} 2}$

La aplicación de las encuestas se hizo de manera virtual. Al final de cada semestre la encuesta se habilitaba en el aula virtual de cada asignatura, en el espacio destinado a la "Información General". En el tablero de anuncios se informaba a los estudiantes sobre la disponibilidad de la encuesta y la importancia que tenía para la Universidad que ellos la diligenciaran.

\section{Variables medidas}

El objetivo de la encuesta era conocer la opinión de los estudiantes con relación a Uso de la plataforma, Interacciones comunicativas, Presentación de los contenidos, Evaluación, y algunas de sus apreciaciones generales sobre la metodología bimodal 
Las 4 variables mencionadas se evaluaron a través de una encuesta que constaba de 22 preguntas, entre estructuradas y semiestructuradas.

\section{Sobre el uso general de la plataforma}

Esta variable tenía como objetivo evaluar el proceso de inducción y la distribución de los espacios en la plataforma con relación a la fácil ubicación de la información por parte de los estudiantes. Este aspecto permite medir, además, la capacidad y el conocimiento que el profesor titular de la asignatura tiene no solo para impartir su clase sino, además, para enseñar a los estudiantes el manejo de la plataforma y el uso que se les puede dar a todas las herramientas que esta tiene para apoyar el proceso enseñanza-aprendizaje.

Los profesores de la Universidad de Medellín reciben semestralmente capacitaciones para el uso avanzado de la plataforma y el diseño de objetos virtuales de aprendizaje. Lo aprendido por ellos debe reflejarse en el manejo que le dan a su curso bimodal.

\section{Sobre la interacción comunicativa en la plataforma}

Aquí se diseñaron 12 preguntas para medir, entre otras cosas, si los espacios de interacción que el profesor diseñó, tales como foros, mensajes y chat fueron suficientes para el proceso de aprendizaje del estudiante. Si el estudiante participó o no de esos espacios, si el profesor lo animó a participar y si las respuestas que obtuvo a través de ellos fueron adecuadas y suficientes para su proceso de aprendizaje.

\section{Sobre la presentación de los contenidos y la evaluación}

En esta variable se diseñaron 5 preguntas para indagar sobre la cantidad de actividades evaluativas, los contenidos de la clase, la calidad del material didáctico, la claridad del profesor para explicar las actividades evaluativas y, por último, se solicitaba a los estudiantes hacer recomendaciones si consideraban que alguno de los aspectos mencionados debía ser mejorado.

\section{Evaluación, y algunas de sus apreciaciones generales sobre la metodología bimodal}

En la última parte de la encuesta se indagó con los estudiantes si era su primera experiencia con la educación bimodal, ventajas y desventajas que le vieron a la educación bimodal y si se matricularían nuevamente en una asignatura bajo esta modalidad de enseñanza.

La tabulación de las respuestas obtenidas en la encuesta fue exportada a Excel y organizada en tablas y gráficos. A continuación se muestran algunos resultados preliminares que se han obtenido durante la medición de la asignatura de Mercadeo I.

En algunos de los resultados analizados hasta el momento se puede observar que, por ejemplo, para la asignatura de Mercadeo I durante el primer semestre del 2011 el 55,8 \% de los estudiantes consideró que el proceso de inducción a la plataforma fue excelente; el 37,2 \%, bueno; el 4,7 \%, regular, y el 2,3 \% respondió que no realizó la inducción.

Este proceso incluyó el reconocimiento de los elementos de la plataforma, la ubicación de los contenidos y recursos, el uso de los elementos del Objeto Virtual de Aprendizaje, 
las normas de Netiqueta que debían utilizar en la comunicación y el uso general de los recursos y actividades.

La misma pregunta en los dos periodos siguientes en la asignatura de Mercadeo I obtuvo resultados que oscilan entre excelente con un $49 \%$ en 2011-2, y $34 \%$, en 2012-1. En los mismos periodos el proceso de inducción fue calificado como bueno por el $40 \%$ y el $64 \%$, respectivamente. Solo un 2,9\% lo calificó como regular en $2011-2$, y $1,7 \%$, en 2012-1. No realizaron el proceso de inducción 6 \% de los estudiantes en el período 2011-2.

Los estudiantes que respondieron que el proceso fue regular argumentan entre otras cosas que el tiempo es corto para aprender a manejar la plataforma UVirtual; otros argumentaron que no estaban matriculados en la asignatura cuando se hizo la inducción.

Otro de los aspectos evaluados durante los tres períodos que se ofreció la asignatura Mercadeo I bajo metodología bimodal fueron las interacciones comunicativas. La plataforma UVirtual cuenta con diferentes herramientas que les permiten a los participantes (estudiantes-profesores) estar en contacto durante todo el semestre. Estas herramientas son chat, foros y mensajes. Con esta pregunta se quiso conocer si los estudiantes usaron las diferentes herramientas de la plataforma para comunicarse con el profesor y si las asesorías académicas a través de estas herramientas fueron puntuales, oportunas, claras y concretas.

Por ejemplo, para los estudiantes de la asignatura Electiva TIC del período académico 2012 _ 2 el $100 \%$ considera que los espacios de comunicación programados por el profesor fueron suficientes para su proceso de aprendizaje. El mismo porcentaje se presenta en el mismo periodo evaluado pero de la asignatura Estética de la Imagen. La asignatura de Mercadeo I no se ofreció bajo metodología bimodal durante el 2012 _ 2

\section{La perspectiva de los profesores frente a la educación bimodal en la Universidad de Medellín}

Para definir cuáles son las características que deben tener las asignaturas bimodales en la Universidad de Medellín y determinar los criterios generales requeridos para su implementación institucional, en concordancia con el modelo pedagógico de la universidad, objetivo de la investigación, se hace necesario indagar por la perspectiva de los profesores durante el proceso de implementación de las asignaturas bimodales. Por tal razón se desarrollaron y aplicaron tres instrumentos de medición a los profesores que cumplieran con todas o con alguna de las siguientes características: a) que hubieran tenido una asignatura bimodal, b) que hubieran pasado por el proceso de capacitación para implementar asignaturas bimodales $\mathrm{o}$, c) que estuvieran en fase piloto de implementación de asignaturas bimodales.

Los instrumentos que se diseñaron y aplicaron fueron: entrevista pos-prueba piloto implementación asignatura bimodal, en las cuales se evaluaron las mismas variables que se evaluaron en la encuesta a los estudiantes. Relatoría del proceso de preparación de 11 profesores de la Universidad adscritos a la Facultad de Comunicación, y entrevista en profundidad a profesores que ya tienen implementadas asignaturas bajo metodología bimodal. 


\section{Resultados}

El proceso de inducción es bien llevado por los profesores, pues la mayoría de los estudiantes ubican su calificación entre excelente y bueno, para las tres asignaturas evaluadas. Sin embargo, los profesores consideran que hay un bache en el proceso de inducción entre los que han tenido experiencias previas con la plataforma de la Universidad y los que llegan a tener ese contacto por primera vez en la inducción.

Profesores y estudiantes consideran que el tiempo de inducción a la plataforma es corto. Para los estudiantes que llegan por primera vez a la plataforma se les dificulta mucho el proceso de inducción. Por lo tanto, se deben hacer dos procesos de inducción: uno para los estudiantes con conocimientos previos y otro para los estudiantes que nunca han tenido experiencia con la plataforma.

Tanto estudiantes como profesores consideran que las tres herramientas que permiten las interacciones comunicativas son suficientes. Se debe trabajar más en el uso de las normas de Netiqueta y la puntualidad en el chat de asesoría con el profesor.

En cuanto a la presentación de los objetos virtuales, tanto los profesores como los estudiantes consideran que tienen todos los elementos necesarios para el aprendizaje, resuelven dudas, son dinámicos, aunque algunos estudiantes los consideran un poco extensos. De las actividades que acompañan a los OVA, las que más aceptación tienen son los juegos de Educaplay, y las que menos, las lecturas.

\section{Conclusiones}

La puntualidad y claridad de las respuestas del profesor a las dudas que los estudiantes envían a través de los foros, mensajes y chat son ampliamente valoradas por los estudiantes; por lo tanto, el profesor debe no solo responder con prontitud, sino que debe hacerlo con la misma eficacia comunicativa como si la respuesta fuera presencial.

Los espacios para las interacciones comunicativas foros, mensajes y chat son suficientes para lograr una comunicación estudiante-profesor y estudiante-estudiante. Para que esta comunicación sea realmente eficaz se debe hacer énfasis tanto a los profesores como a los estudiantes, de la importancia de su uso. Esto se debe hacer en los procesos de inducción.

Los chat de asesoría con el profesor deben ser programados en el horario regular de clase, para disminuir la baja participación en los mismos. En las encuestas, los estudiantes que no participaron del chat argumentaron estar cumpliendo labores de otras asignaturas.

Los espacios de discusión académica como los foros sobre temas específicos de clase son fundamentales porque desarrollan en los estudiantes, la capacidad de expresar y defender sus ideas, contra-argumentar, mejoran la comprensión lectora y fomentan el autoaprendizaje.

No existe consenso respecto al número de actividades de aprendizaje y calificables que deben existir en las asignaturas bimodales. El número de actividades y porcentajes 
varía de una asignatura a otra. Hasta el momento en la Universidad de Medellín, las asignaturas bimodales están cobijadas por el reglamento de las asignaturas presenciales; por lo tanto, sí hay un límite de actividades evaluativas y porcentajes de calificación que se destinan a estas.

\section{Bibliografía}

Arango Vásquez, S. I., \& Vásquez Lopera, C. P. (2010). Ponencia: Educación virtual y TIC - Un caso de innovación en la Universidad de Medellín". Encuentro de Ciencias Sociales y Humanas. Medellín.

Bunge, M. (1997). Ciencia, técnica y desarrollo. Uruguay: Editorial Sudamericana.

Castillo Torres, M. (2013). Lineamientos de calidad para la verificación de las condiciones de calidad de los programas virtuales y a distancia. Bogotá: República de Colombia convenio Andrés Bello.

Castro Gil, M. A., Colmenar Santos, A., Losada de Dios, P., E Peire Arroba, J. (2003). Diseño y desarrollo Multimedia. Sistemas, Imagen, Sonido y Video. Madrid: RA-MA editorial.

Colombia aprende. La red del conocimiento. (s. f.). Obtenido de http://www.colombiaaprende.edu.co/html/ directivos/1598/article-172365.html

Colombiaaprende. (s.f.). Obtenido de http://www.colombiaaprende.edu.co/html/directivos/1598/ article-99543.html

Expoelearning. (Mayo de 2013). Expoelearning Congreso Internacional y Feria Profesional. Obtenido de http:// www.expoelearning.com/wp-content/uploads/2014/09/NP2 165crecimiento23.05.13.pdf

Fontela, Hellers, Mann, Podlesker, \& Subotovsky. (2004). Contenidos de e-learning. Tendencias, procesos, recomendaciones. Buenos Aires: Ediciones AXG Tecnonexo.

García Aretio, L. (Junio de 1999). Historia de la Educación a Distancia. Ried - Revista Iberoamericana de Educación a Distancia, 2(1), 8-27. doi:http://dx.doi.org/10.5944/ried.2.1.2084

Gobierno de España, Ministerio de industria, turismo y comercio, E Ministerio de educación. (5 de febrero de 2013). Formación en didactica TIC. Obtenido de http://didacticatic.educacontic.es/sites/ default/files/tree/183/es/ver/index.html

González Agudelo, E. (2006). Sobre la hermenéutica o acerca de las múltiples lecturas de lo real. Medellín: Sello Editorial Universidad de Medellín.

González Guerrero, K., Padilla Beltrán, J. E., E Rincón Caballero, D. A. (2012). El docente en el contexto b-learning. Bogotá: Universidad Militar Nueva Granada.

Guzmán Hernández, T. d. (Enero-Marzo de 2008). La universidad ante los retos que plantea la sociedad del conocimiento, ante los avances tecnológicos en las TIC. Revista Tecnología en marcha, 21-1, 4-15. Obtenido de http://www.tec.ac.cr/sitios/Nicerrectoria/vie/editorial_tecnologica/Revista_Tecnologia_ Marcha/pdf/tecnologia_marcha_21-1/4-15.pdf

Hassan Montero, Y., E Martín Fernández, F. J. (s.f.). No solo usabilidad: revista sobre personas, diseño y tecnología. Obtenido de http://www.nosolousabilidad.com/articulos/escritura_hipertextual.htm

Hernández Sampieri, R., Fernández Collado, C., E Baptista Lucio, P. (1997). Metodología de la Investigación. México: McGraw Hill. 
Holzschlag, M. (2002). Color para sitios Web. México: McGraw-Hill Interamericana Editores, S.A.

Lara Navarra, P., Saigí, F., \& Duart, J. (2004). El posicionamiento en Internet de Instituciones culturales, científicas y educativas : 5 al 7 de mayo de 2004, organizadas por la Universidad de Alicante, Fundación Biblioteca Virtual Miguel de Cervantes y Casa de las Américas. Posicionamiento web de contenidos en e-learning. Accesibilidad y usabilidad como instrumento de competitividad y calidad (pág. 20). Alicante: Biblioteca Virtual Miguel de Cervantes, 2004.

Malhotra, N. (2008). Investigación de mercados (5 ed.). México: Pearson Educación.

Marí Sáez, V. (2006). Jóvenes, tecnologías y el lenguaje de los vínculos. Revista científica de comunicación y educación, 27, 113-116.

Martí Arias, J. A. (Abril - Junio de 2009). Aprendizaje mezclado (B-Learning). Revista Universidad Eafit, 45(154), 70-77. Recuperado el 2012

Massoud, A., Iqbal, U., Stockley, D., E Noureldin, A. (Noviembre de 2011). Using Blended learning to Foster education. Transformative Dialogues, 5(Issue 2).

MEN. (19 de Julio de 2009). Ministerio de Educación Nacional, República de Colombia. Obtenido de http:// www.mineducacion.gov.co/1621/article-196492.html

MEN. (s.f.). Colombia Aprende. Obtenido de http://www.colombiaaprende.edu.co/html/directivos/1598/ article-172369.html

MEN, M. R. (2014). MinEducación. Obtenido de http://www.mineducacion.gov.co/1621/articles-229430_ archivo_pdf_decreto1295.pdf

MEN, M. R. (2014). Revolución Educativa Colombia Aprende. Obtenido de http://www.mineducacion.gov. $\mathrm{co} / 1621 /$ article-217768.html

Nielsen, J. (2000). Usabilidad. Diseño de sitios Web. Madrid: Prentice Hall.

Ocak, M. (2010). Blend or not to blend: a study investigating faculty members' perceptions of blended teaching. World Journal on Educational Technology, 2(issue 3), 196-210.

Poulin, R. (2012). El lenguaje del diseño gráfico. Conocimiento y aplicación práctica de los principios fundamentales del diseño. Barcelona: Promopress.

Revuelta Domínguez, F. I., E Pérez Sánchez, L. (2009). Interactividad en los entornos de formación on-line. Barcelona: UOC.

Sánchez Upegui, A. A., Puerta Gil, C. A., \& Sánchez Ceballos, L. M. (2010). Manual de comunicación en ambientes educativos virtuales. Medellín: Fundación Universitaria Católica del Norte.

Silva, M. (2005). Educación interactiva. Enseñanza y aprendizaje presencial y on-line. Barcelona: Gedisa.

Tamayo, E Tamayo, M. (2002). El proceso de la investigación científica (4 ed.). México: Noriega Editores.

UNAD, EAN, CEIPA, ACESAD, \& Red Colombia de IES con Programas a Distancia. (2014). Bases del proyecto de política pública para el fomento y desarrollo de la modalidad de educación a distancia en Colombia. Documento de trabajo 3. a. Versión. Bogotá. 
UNIAJC, C. A. (2013). Institución Universitaria Antonio José Camacho. Recuperado el 2014, de http://www. uniajc.edu.co/documentos/normatividad/resoluciones/No.\%20019.pdf

Unigarro Gutiérrez, M. A. (2004). Educación virtual: Encuentro formativo en el ciberespacio (2 ed.). Bucaramanga: UNAB.

Universidad de Medellín. (2014). Universidad de Medellín. Educación Virtual y TIC: Formación docente FAVA. Recuperado el 2015, de www.udem.edu.co/index.php/formacion-docente/fava?highlight=WyJtb2RlbG8iLCJwZWRhZ1x1MDBmM2dpY28iLCJtb2RlbG8gcGVkYWdcdTAwZjNnaWNvIl0=

Vásquez Lopera, C. P., E Arango Vásquez, S. I. (2010). Capítulo 7: Interacciones comunicativas en un Entorno Virtual de Aprendizaje (EVA). En Pensar la comunicación - Reflexiones y resultados de investigación, Tomo II (págs. 129-146). Medellín: Sello Editorial Universidad de Medellín.

Williams, R., E Tollett, J. (2006). Diseño Web. Madrid: Ediciones Anaya Multimedia.

Yábar, J. M., E Barbarà, P. L. (1999). La Universidad Autónoma de Barcelona: El camino hacia una universidad bimodal en el marco de las Tecnologías de la Información y la Comunicación. (Educar, Ed.) Educar(25), 113-118. Obtenido de http://educar.uab.cat/article/view/298

Yábar, J. M., Barbarà, P. L., Añaños, E., Recoder, M. J., \& Hernández, J. (s. f.). Ponencia: La Universidad Autónoma de Barcelona: una universidad para el siglo XXI. Barcelona. Obtenido de http://reposital. cuaed.unam.mx:8080/jspui/bitstream/123456789/2392/1/01_43.pdf 